\title{
PORPHYROMONAS GINGIVALIS IN CORONARY ATHEROMA AND SUBGINGIVAL PLAQUE - A CLINICAL AND MICROBIOLOGICAL STUDY
}

Col S K Rath ${ }^{1}$, Col S Sen², Maj Nitin Gupta ${ }^{3}$, Mukesh4, Brig S K Roy ${ }^{5}$

\section{HOW TO CITE THIS ARTICLE:}

Col S K Rath, Col S Sen, Maj Nitin Gupta, Mukesh, Brig S K Roy. "Porphyromonas Gingivalis in Coronary Atheroma and Subgingival Plaque - A Clinical and Microbiological Study". Journal of Evolution of Medical and Dental Sciences 2014; Vol. 3, Issue 03, January 20; Page: 551-558, DOI:10.14260/jemds/2014/1864

ABSTRACT : BACKGROUND: There has been increasing attention paid in recent years to the possibility that oral bacterial infection, particularly periodontal disease may influence the initiation and or progression of systemic diseases. These studies confirm the observation that heart disease is the most commonly found systemic condition in patients with periodontal disease. Moreover, the literature has also highlighted substantial evidence indicating the presence of gram negative periodontal pathogens in atheromatous plaques. AIMS :-The present study intends to investigate the possible association between periodontal health and coronary artery disease by evaluating periodontal status, association between the periodontal plaque and coronary atheromatous plaques for presence of P.gingivalis. SETTINGS AND DESIGN: A case control study was designed with 07 patients who had underwent coronary endarterectomy for CVD and 28 controls. The periodontal examination for cases was performed one day before vascular surgery and the controls were clinically examined. METHODS AND MATERIAL : The atheromatous plaque sample collected during endarterectomy and the Intraoral plaque samples were subjected to PCR for identification of P.gingivalis. The presence of periodontal bacteria DNA in coronary atheromatous plaques and subgingival plaque samples of the same patients was confirmed by this study. STATISTICAL ANALYSIS USED : Means and proportions for personal characters, major risk factors and clinical parameters were calculated for both the groups. The significance of any difference in means was tested by using "Students t test", and the significance of any difference in proportions was tested by using Dunn- Sidak Adjusted p Value. RESULTS : During the microbial analysis of plaque samples by PCR in group A it was seen that Porphyromonas gingivalis in $100 \%$ of the samples. Microbial analysis of endarterectomy samples by PCR in group A shows that Porphyromonas gingivalis was found in $71.43 \%$ of endarterectomy samples. Porphyromonas gingivalis was present in all the plaque samples and 5 atheroma samples of Group A. CONCLUSIONS: A correlation was established between putative bacteria contributing to atheromatous plaques and species associated with periodontal disease. One particularly important study to be carried out is the investigation of a possible clinically meaningful reduction in coronary heart disease resulting from the prevention or treatment of periodontal disease.

\section{KEY WORDS: PORPHYROMONAS GINGIVALIS, PCR, ATHEROMATOUS PLAQUE}

INTRODUCTION: Periodontitis is a multifactorial disease, with microbial dental plaque as the initiator of periodontal disease. Recent studies have proven that periodontal disease can produce numerous changes in systemic health with changes in the blood chemistry with a rise in inflammatory mediators, proteins and lipids in the serum.1,2 There has been increasing attention paid in recent years to the possibility that oral bacterial infection, particularly periodontal disease may influence the initiation and or progression of systemic diseases. Periodontitis has been proposed as having an etiological or modulating role in Cardiovascular and Cerebrovascular disease, Diabetes, Respiratory diseases, adverse pregnancy outcome, and rheumatoid arthritis. ${ }^{3,4}$ Studies of 
different populations have indicated that atherosclerosis and thrombo-embolic events may be related to oral infections. Thus, periodontitis shares a series of features with cardiovascular diseases, such as a higher incidence in adult males, smokers, diabetics, and individuals with stress and/or a low socioeconomic level.

Studies conducted on different populations have suggested that atherosclerosis and the occurrence of acute myocardial infarction could be linked with chronic oral infections. ${ }^{5}$ These studies confirm the observation that heart disease is the most commonly found systemic condition in patients with periodontal disease. Moreover, the literature has also highlighted substantial evidence indicating the presence of gram negative periodontal pathogens in atheromatous plaques. ${ }^{6}$ Systemic low grade infections with their moderate acute phase responses may accelerate the formation of atheromatous plaque with consequent increased risk of future cardiovascular events. ${ }^{7}$

Considerable evidence supports a plausible set of mechanisms by which periodontopathic bacteria may directly or indirectly contribute to cardiovascular disease, such as blood platelet aggregation, enhanced low-density cholesterol and lipoprotein deposition in the artery walls, invasion of cardiac and carotid endothelium, and the high level of inflammatory mediators in the circulation and tissues.

In recent years, studies have implicated Aggregatibacter actinomycetemcomitans, Prevotella intermedia, Porphyromonas gingivalis and Tannerella forsythia in connective tissue attachment loss and periodontal inflammation.

The present study intends to investigate the possible association between periodontal health and coronary artery disease by evaluating periodontal status, association between the periodontal plaque and coronary atheromatous plaques for presence of P.gingivalis.

MATERIALS AND METHODS: A case control study was designed in the Department of Dental Surgery and conducted in the Coronary Care Unit (CCU) of the Department of Cardiothoracic Surgery. Seven patients undergoing coronary end arterectomy for CVD in the Department of Cardiothoracic Surgery were selected as cases and twenty eight controls subjects randomly selected from the patients attending Dental out Patient Department of Dental Surgery. The necessary permission and ethical clearance was obtained. All subjects were verbally informed and a duly signed written informed consent was obtained from all the patients prior to their enrolment.

Selection Criteria: Following inclusion/exclusion criteria were followed for the study:

\section{Inclusion criteria:}

1. Cases were the patients undergoing coronary end arterctomy for CVD.

2. Controls were subjects with healthy periodontium without CVD randomly selected from patients attending Dental OPD.

3. Age group between 40 and 60 years.

4. Presence of at least 14 natural teeth.

5. No history of scaling/root planing or systemic antibiotic therapy in the previous 6 months.

6. No history of other systemic diseases.

\section{Exclusion criteria for cases}

1. Edentulous patients.

2. Patients who had received periodontal treatment within the past six months.

3. Patients in whom oral examination was considered unsafe or contraindicated. 
4. Patients who were using immunosuppressive drugs or were under chemotherapy.

5. Pregnant women.

6. Patients who could not co-operate in oral examination.

7. Antibiotic therapy in the last one week.

8. Patients from whom a written informed consent could not be obtained.

After subject enrollment by an examiner, the following groups were assigned.

Group A (Cases): Plaque sample collected from periodontal pocket of the cases, atheroma sample collected after end arterctomy and were later subjected to identification of periodontal pathogens by sampling DNA extract and amplification by Polymerase Chain Reaction (PCR).

Group B (Controls): 1:4 cases were selected as controls by randomization. The plaque sample from gingival sulcus collected by a sterile paper point in the pocket later subjected to identification of periodontal pathogens by sampling DNA extract and amplification by PCR.

Periodontal Examination: The periodontal examination for cases was performed one day before vascular surgery in cardiothoracic ward. The controls were clinically examined in Division of Periodontics in Department of Dental surgery. Clinical data were recorded using guidelines as described below (Fig 1 \& 2).The clinical parameters included Pocket Depth (PD), Clinical Attachment Level (CAL), Periodontal Pocket Bleeding Index (PPBI). ${ }^{8}$

Microbiological Examination: The atheromatous plaque sample of 2-10 mm long collected during end arterectomy were harvested and specimens obtained were placed in $10 \mathrm{ml}$ of saline solution in sterile vials. The Intraoral plaque samples collected from the same patient in group A and from the control subjects during periodontal examination were also placed in $10 \mathrm{ml}$ saline solution. All samples were immediately frozen in a bath of liquid nitrogen and stored at $-80^{\circ} \mathrm{C}$ until DNA preparation in the Laboratory of Microbiology and Virology.

Step: - 1: All the samples were subjected to identification for Porphyromonas gingivalis by sampling DNA extract and amplification by PCR. Approximately $100 \mathrm{mg}$ of tissues was harvested from the atherosclerotic plaques and Intra oral plaque then homogenized and subjected to DNA extraction. DNA was extracted by the Qiagen Kit method. After this the eluted DNA was stored at $-20^{\circ} \mathrm{C}$ (Fig 1).

Step: - 2: The whole genomic DNA extracts were used as templates in a PCR technique using the universal primer (Fig 2) that targeted the 16S rRNA gene. The primers used were presented in Table-1. In the second round 40 cycles of PCR was run in thermo cycler with appropriate cycling condition.

\begin{tabular}{|c|c|c|}
\hline Microorganism & Nucleotide Sequence & No. of Base pairs(bp) \\
\hline P. gingivalis (PgF) & 5'TGTAGATGACTGATGGTGAAAACC3' & 197bp \\
\hline Conserve Reverse (ConR) & 5'ACGTCATCCCCACCTTCCTC-3' & \\
\hline & Table- 1 (PRIMER)
\end{tabular}

Step: - 3: Gel documentation for multiplex PCR: The amplified amplicon of second round PCR was demonstrated by using 2\% agarose gel electrophoresis (Fig 3). By using 100 bp molecular weight marker the periodontal pathogen were identified based on the bp molecular weight (Fig 4).

Statistical analysis: The clinical and microbiological data collected was tabulated and was subjected to statistical analysis. The independent variables included age, gender, 
education, socioeconomic status, marital status, medical status, smoking status, number of missing teeth, pocket depth; number of teeth with CAL $>3 \mathrm{~mm}, \%$ sites with BOP and CAL were used to quantify the type of disease.

Means and proportions for personal characters, major risk factors and clinical parameters were calculated for both the groups. The significance of any difference in means was tested by using "Students t test", and the significance of any difference in proportions was tested by using Dunn- Sidak Adjusted p Value.

Means and proportions for personal characters, major risk factors and clinical parameters were calculated for both the groups. The significance of any difference in means was tested by using unpaired Students " $t$ " test.

RESULTS: Intra group comparisons of demographic parameters among cases i.e. patients undergoing coronary end arterectomy showed no statistically significant difference between the cases with respect to age, sex, education, socioeconomic and smoking status with the $\mathrm{p}$ value of 1.000 for all variables and Dunn-Sidak adjusted $p$ value of $1.000,0.959,1.000,0.989,1.000$ respectively (Table 2).

No statistically significant difference was observed in any of the periodontal parameters including PD, CAL, PBBI, When the intra group comparisons were done with Dunn-Sidak adjusted $\mathrm{p}$ value of $1.000,0.861,1.000,0.689$ respectively (Table 3 ).

The result of intra group comparisons of microbiological examination by PCR of subgingival plaque samples and coronary end arterectomy atheroma samples for identification of Porphyromonas gingivalis showed statistically significant difference. (Table 4).

During the microbial analysis of plaque samples by PCR in group A it was seen that Porphyromonas gingivalis in $100 \%$ of the samples. Microbial analysis of end arterectomy samples by PCR in group A show that Porphyromonas gingivalis was found in $71.43 \%$ of end arterectomy samples. Porphyromonas gingivalis was present in all the plaque samples and 5 atheroma samples of Group A (Table 5, 6).

DISCUSSION: Since the sample size of the cases was small, the intra and inter group comparisons of periodontal parameters were statistically performed by the help of Dunn-Sidak Adjusted 'p' Value. The first coronary end arterectomy was done by Baily et al. (1957). Because of increased morbidity and mortality, the procedure of coronary end arterectomy had lost its importance. 9, 10 WD Jhonson and DA Cooley who performed a large series of coronary end arterectomy revealed that there is an increase in surgical risk and good long term effect in selected group of patient. 11 We could get a small number of end artrectomy samples after surgical procedure from Department of Cardiothoracic Surgery as it is a rare procedure and potential cause for surgical complications.

The subgingival samples of all the seven cases were positive for porphyromonas gingivalis, which was also seen in significant number of atheromatous plaque sample. Thus a correlation may be established between coronary heart disease and periodontal disease. The presence of periodontal bacteria in atheromatous plaques and periodontal samples of the same cases supports the potential role of this periodontopathogenic bacterial species in some steps of the atherogenesis process or as a contributor of a different mechanism that worsens this disease. The link found in this study supports the concept that there may be a true inflammatory link between atherogenesis and periodontitis. The data of this study were consistent with those reported by Haraszthy et al ${ }^{12}$ (PCR- 
amplified 16S rDNA and DNA species-specific probes, 30\% positive for $\mathrm{T}$. forsythia, $26 \%$ for P.gingivalis, 14\% for P. intermedia), Ishiara et al ${ }^{13}$ (PCR-amplified $16 \mathrm{~S}$ rRNA, $21.6 \%$ positive for P. gingivalis, 5.9\% for T. forsythia) and ZHANG Y M et al. ${ }^{14}$

Periodontal patients harboring Porphyromonas gingivalis have shown higher levels of lipids, low-density lipoprotein and total cholesterol in the bloodstream, which seem to be associated with the development of atheromatous plaques. On the other hand, the high prevalence of Gram-negative oral bacteria in atherosclerotic tissues may induce the secretion of several cytokines associated with the development of cardiovascular diseases by their LPS. 15

A direct causal relationship between periodontitis and atherosclerotic CVD is not established. Multiple studies, however, support two biologically plausible mechanisms:

1. Moderate to severe periodontitis increases the level of systemic inflammation, a characteristic of all chronic inflammatory diseases, and periodontitis has been associated with increased systemic inflammation as measured by hs CRP and other biomarkers. Treatment of moderate to severe periodontitis sufficient to reduce clinical signs of the disease also decreases the level of systemic inflammatory mediators. ${ }^{16,17}$

2. In untreated periodontitis, gram-negative bacteria may be found in periodontal pockets surrounding each diseased tooth and in approximation to ulcerated epithelium, and bacterial species found predominantly in the periodontal pockets also have been found in atheroma. ${ }^{18}$

Due to the sensitivity of PCR for detecting microbial DNA, sample contamination as a result of bacteremia or handling of samples is a cause of concern. ${ }^{[19]}$ However, because of the high level of microbial DNA observed in most of the atheromatous plaques, it is unlikely that these patients suffered accidental contamination; instead, it suggests a stable and lasting microbial colonization of atheromas.

However the current study has a few limitations. Firstly, the sample sizes were too small taking into account the number of end arterectomy being too small because of mortality and morbidity associated with it. Secondly, quantitative analysis of the identified bacteria by PCR technique was a very sensitive procedure. The PCR technique could identify the type of bacteria present in both subgingival sample and atheroma samples but failed to quantify them. A real time PCR technique would have been more appropriate for quantitative analysis of bacteria.

CONCLUSION: The presence of periodontal bacteria in atheromatous plaques and periodontal samples of the same cases supports the potential role of this periodontopathogenic bacterial species in some steps of the atherogenesis process or as a contributor of a different mechanism that worsens this disease. The link found in this study supports the concept that there may be a true inflammatory link between atherogenesis and periodontitis. Thus, taken together with our results, this suggests that Porphyromonas gingivalis reach the bloodstream, playing a direct or indirect role in the pathogenesis of cardiovascular diseases. The results shown here reinforce the importance of periodontal bacteria as a possible contributing factor in the development of cardiovascular diseases. Knowledge of the micro-organisms present in atheromas from patients with chronic periodontitis is relevant in the prevention and treatment of cardiovascular infections that appear to be produced, in part, by these oral periodontopathic bacteria.

However, legitimate concerns have arisen about the nature of this relationship. Since even a moderate risk contributed by periodontal disease to heart disease could lead to significant morbidity and mortality, it is imperative that further studies be conducted to evaluate this relationship. One 
particularly important study to be carried out is the investigation of a possible clinically meaningful reduction in coronary heart disease resulting from the prevention or treatment of periodontal disease.

\section{REFERENCES:}

1. James D B, Offenbacher S. Systemic effects of periodontitis: epidemiology of periodontitis and cardiovascular disease. J Periodontol 2005; 76:2089-2100.

2. Loos B G. Systemic markers of inflammation in periodontitis. J Periodontol 2005; 76:2106-15.

3. Mattila K J, Pussinen PJ, Paju S. Dental infections and cardiovascular diseases- A review. J Periodontol 2005; 76: 2085- 88.

4. Dissick A, Redman RS, Jones M et al. Association of Periodontitis with Rheumatoid Arthritis: A Pilot Study J Periodontol 2010; 81: 223-30.

5. Mattila KJ, Nieminen MS, Valtonen V. Association between dental health and acute myocardial infarction. Br Med J. 1989; 298: 779-81.

6. Haraszthy VI, Zambon JJ, Trevisan M, Zeid M, Genco RJ. Identification of periodontal pathogens in atheromatous plaques. J Periodontol. 2000; 71:1515-61.

7. Danesh J, Collins R, Appleby P, Peto R. Association of fibrinogen, C-reactive protein, albumin, or leukocyte count with coronary heart disease: meta-analyses of prospective studies. JAMA 1998; 279: 1477-82.

8. Hujoel PP, Orangsholt M, Spiekman C et al. Periodontal disease and coronary heart disease. JAMA 2000; 284: 1406-10.

9. Effler DB, Groves LK, Sones FM. Endarterectomy in the treatment of coronary artery disease. J Thorac Cardiovas Surg 1996; 47:98-108.

10. Johnson WD, Brenowitz JB, Karver KL. Surgery for different coronary disease. Cardiology 1986; 3:35-8.

11. Padhy K, Narshimam SBR. Murthy RC et al. Coronary endarterectomy for diffuse extensive coronary artery disease. Ind J Thorac Cardiovasc Surg, 2005; 21:251-5.

12. Haraszthy VI, Zambon JJ, Trevisan M, Zeid M, Genco RJ. Identification of periodontal pathogens in atheromatous plaques. J Periodontol 2000; 71: 1554-60.

13. Ishihara K, Nabuchi A, Ito R, Miyachi K, Kuramitsu HK, Okuda K. Correlation between detection rates of periodontopathic bacterial DNA in coronary stenotic artery plaque and in dental plaque samples. J Clin Microbiol 2004; 42: 1313-5.

14. Zhang Y M, Zhong L J, Liang P, Liu H, La-ti MU, Si-ka-er AI. Relationship between microorganisms in coronary atheromatous plaques and periodontal pathogenic bacteria. Chinese Medical Journal 2008; 121(16):1595-7.

15. Fiehn NE, Larsen T, Christiansen N, Holmstrup P, SchroederT V. Identification of periodontal pathogens in atherosclerotic vessels. J Periodontol 2005; 76: 731-6.

16. Bahekar AA, Singh S, Saha S, Molnar J, Arora R. The prevalence and incidence of coronary heart disease is significantly increased in periodontitis: A meta-analysis. Am Heart J 2007; 154:8307.

17. Paraskevas S, Huizinga JD, Loos BG. A systematic review and meta-analyses on C-reactive protein in relation to periodontitis. J Clin Periodontol 2008; 35: 277-90.

18. Tonetti MS, D'Aiuto F, Nibali L, et al. Treatment of periodontitis and endothelial function. $\mathrm{N}$ Engl J Med 2007; 356:911-20. 


\section{ORIGINAL ARTICLE}

19. Sanz M, Lau L, Herrera D, Morillo J M, Silva A. Methods of detection of Actinobacillus actinomycetemcomitans, Porphyromonas gingivalis and Tannerella forsythensis in periodontal microbiology, with special emphasis on advanced molecular techniques: a review. J Clin Periodontol 2004; 31: 1034-47.

\begin{tabular}{|l|c|c|c|}
\hline \multicolumn{1}{|c|}{ VARIABLES } & df & p value & $\begin{array}{c}\text { Dunn-Sidak } \\
\text { Adjusted p Value }\end{array}$ \\
\hline AGE & 6 & 1.000 & 1.000 \\
\hline SEX & 6 & 1.000 & 0.959 \\
\hline EDUCATION & 6 & 1.000 & 1.000 \\
\hline SOCIOECONOMIC STATUS & 6 & 1.000 & 0.989 \\
\hline SMOKING & 6 & 1.000 & 1.000 \\
\hline
\end{tabular}

TABLE -1: INTRA GROUP COMPARISONS OF DEMOGRAPHIC DATA AMONG CASES

\begin{tabular}{|c|c|c|c|}
\hline VARIABLES & df & p value & $\begin{array}{c}\text { Dunn-Sidak } \\
\text { Adjusted p Value }\end{array}$ \\
\hline PDmm & 6 & 1.000 & 1.000 \\
\hline CALmm & 6 & 0.867 & 0.861 \\
\hline PPBI & 6 & 1.000 & 1.000 \\
\hline PI & 6 & 0.689 & 0.689 \\
\hline
\end{tabular}

TABLE 2: INTRA GROUP COMPARISONS OF PERIODONTAL EXAMINATION PARAMETERS AMONG CASES (Group A)

\begin{tabular}{|c|c|c|c|}
\hline VARIABLES & df & p value & $\begin{array}{c}\text { Dunn-Sidak } \\
\text { Adjusted p Value }\end{array}$ \\
\hline P g & 27 & 0.001 & 0.001 \\
\hline
\end{tabular}

TABLE 3: INTER GROUP COMPARISONS OF MICROBIOLOGICAL EXAMINATION BY PCR BETWEEN SUBGINGIVAL PLAQUE SAMPLES OF CASES AND CONTROLS (Group A and B)

* Values $>0.05$ are not significant

The Dunn- Šidák correction is derived by assuming that the individual tests are independent. The Dunn- Šidák is a safeguard against multiple tests of statistical significance on the same data falsely giving the appearance of significance, as 1 out of every 20 hypothesis-tests is expected to be significant at the $\alpha=0.05$ level purely due to chance.

\#String characters are converted to numeric characters

\begin{tabular}{|c|c|c|}
\hline Micro organisms & No. of cases seen & Percentage \\
\hline P g & 7 & 100 \\
\hline TABLE 4: MICROBIAL ANALYSIS BY PCR IN CASES, Group A (PLAQUE SAMPLES) \\
\hline Micro organisms & No. of cases seen & Percentage \\
\hline P g & 5 & 71.43 \\
\hline TABLE 5: MICROBIAL ANALYSIS BY PCR IN CASES GROUP A (ENDARTERECTOMY SAMPLES) \\
\hline
\end{tabular}

\begin{tabular}{|c|c|c|}
\hline Micro organisms & No. of cases seen & Percentage \\
\hline P g & 2 & 7.14 \\
\hline
\end{tabular}

TABLE 6: MICROBIAL ANALYSIS BY PCR IN CONTROLS (PLAQUE SAMPLES) 


\section{ORIGINAL ARTICLE}
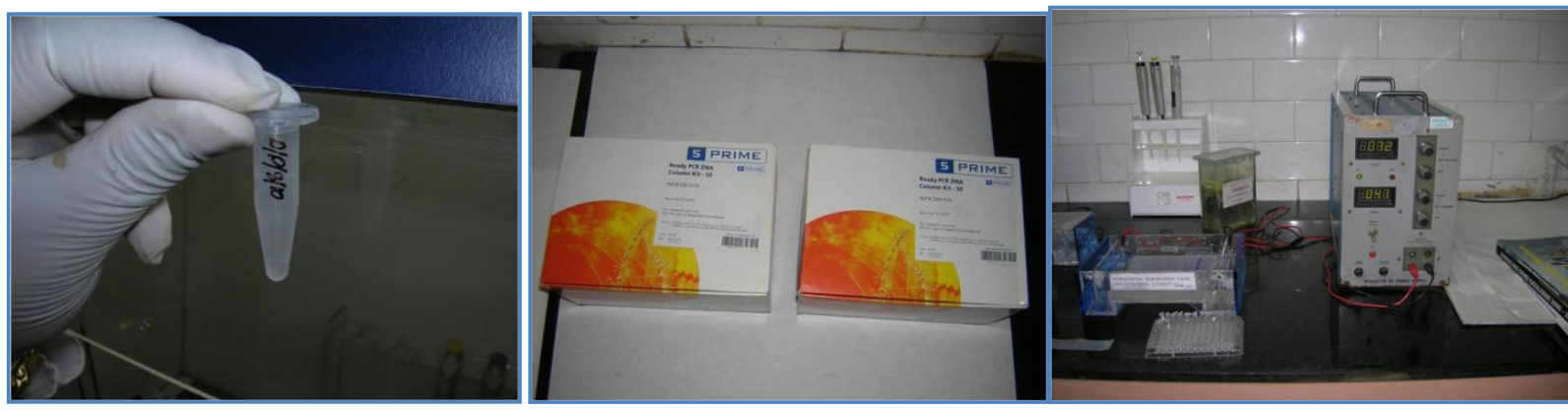

Fig. 1: Extracted DNA sample

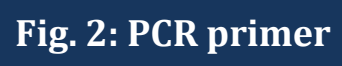

Fig. 3: Gel electrophoresis
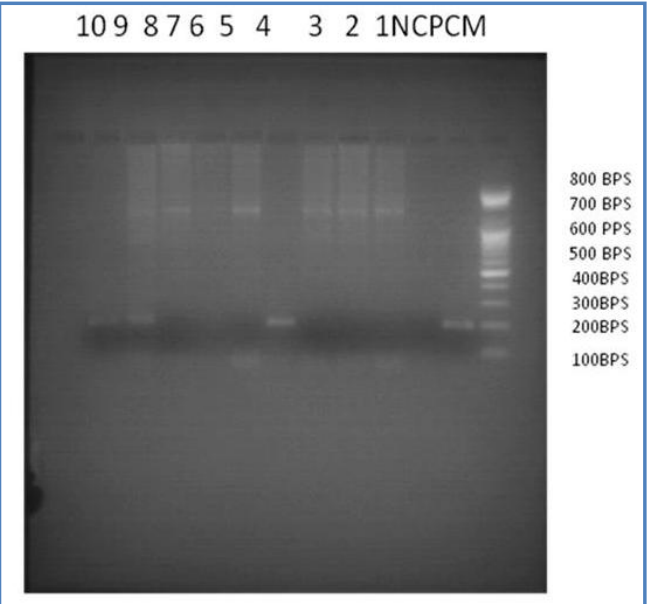

Fig. 4: Amplified product of multiplex PCR 197 bps of band in P gingivalis

\section{PARTICULARS OF CONTRIBUTORS:}

1. Senior SPL, Department of Periodontology, Army Dental Centre, R \& R Hospital, Delhi, CANTT.

2. Senior SPL, Department of Periodontology, Army Hospital, R \& R Hospital, Delhi, CANTT.

3. Dental Officer, Department of Periodontology, CMDC (EC).

4. Senior Resident, Department of Periodontology, Army Dental Centre, R \& R, Delhi, Cantt.

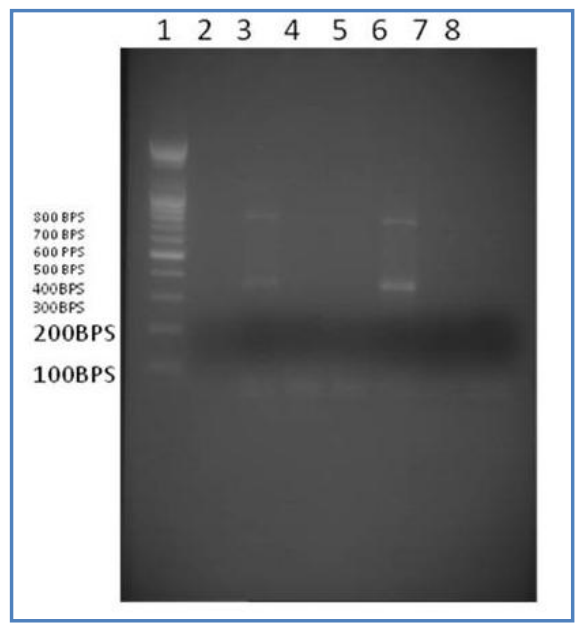

Fig. 5: Amplified product of multiplex PCR band in $3 \& 6$ wells showing Aa 360 bps and Tf 745

5. Commandant, Department of Periodontology, Army Dental Centre, R \& R Hospital, Delhi, CANTT.

\section{NAME ADDRESS EMAIL ID OF THE CORRESPONDING AUTHOR:}

Dr. Col S.K. Rath,

Army Dental Centre,

Delhi, Cantt,

New Delhi - 110010.

Email - drrathfamily@yahoo.com

Date of Submission: 13/12/2013.

Date of Peer Review: 14/12/2013.

Date of Acceptance: 07/01/2014.

Date of Publishing: 14/01/2014. 